\title{
An AUTONOMOUS STAR IDENTIFICATION ALGORITHM BASED ON THE DIRECTED CIRCULARITY PATTERN
}

\author{
Junfeng Xie ${ }^{\mathrm{a}, *}$, Xinming Tang ${ }^{\mathrm{a}}$, Wanshou Jiang ${ }^{\mathrm{b}}$, Xingke Fu ${ }^{\mathrm{a}}$ \\ a Satellite Surveying and Mapping Application Centre, Lianhuachi West Road 28,Beijing, P.R.China (junfengxie, \\ tangxinming99)@gmail.com, fxk@sasmac.cn \\ b State Key Laboratory of Information Engineering in Surveying, Mapping and Remote Sensing, Wuhan University, \\ Luoyu Road 129, Wuhan, P.R.China, jws@lmars.whu.edu.cn
}

\section{Commission I, WG I/5}

KEY WORDS: Angular distance, Matching probability, Matching group, Directed circularity pattern, The longest matching chain

\begin{abstract}
:
The accuracy of the angular distance may decrease due to lots of factors, such as the parameters of the stellar camera aren't calibrated on-orbit, or the location accuracy of the star image points is low, and so on, which can cause the low success rates of star identification. A robust directed circularity pattern algorithm is proposed in this paper, which is developed on basis of the matching probability algorithm. The improved algorithm retains the matching probability strategy to identify master star, and constructs a directed circularity pattern with the adjacent stars for unitary matching. The candidate matching group which has the longest chain will be selected as the final result. Simulation experiments indicate that the improved algorithm has high successful identification and reliability etc, compared with the original algorithm. The experiments with real data are used to verify it.
\end{abstract}

\section{INTRODUCTION}

With the increasement of the resolution of the satellite images, how to quickly acquire accurate location information of image has been an important research for the remote sensing technique. At present, without the information of the ground control points, directly acquiring location information of the object in the image based on the orbit position and attitude information of the satellite has become a development trend of the satellite application. Nevertheless, high directly positioning accuracy inevitable require high attitude accuracy. Compared with other attitude sensors, as the most accurate sensor, the star sensor has many advantages such as no drift and providing three axis attitudes information, etc.

In the process of the attitude determination by star sensor, the stars in the inertia system are considered as references, which are imaged by the CCD camera, and a series of procedures will be implemented [Leitmann,1994], which involves star acquirement[Samaan,2002;Quine,2007;Rufino,2003;Zheng,200 5] ,star identification [padgett,etc,1997; Mortari.etc,2001, Quine,etc.1995; Liebe, 1995; Douma,etc.,1997], and attitude estimation [Shuster,1981;Markley,etc.,1993; Mortari,1998]. Star identification is the most crucial step, which is related to the efficiency and reliability of the software system.

According to the star recognition state in the flight process of satellite, star recognition can be divided into two stages: autonomous and tracking identification modes. Since the autonomous star recognition doesn't need the initial attitude information provided by other sensors (such as gyroscopes etc), it has the higher research values. At present, most star recognition algorithms are designed for autonomous recognition[padgett,etc,1997;Mortari.,etc,2001,Quine ,etc.1995; Liebe.,1995;Douma,.etc,1997;Anderson,1991;SILANI,etc,2006 ]. According to different design mode, there algorithms can be divided into two classes[Padgett,etc.,1997;SILANI,etc.,2006]: one class is to find a subgraph constructed by star image points that is isomorphic to the designed graph constructed by the catalogued stars, and the matching element includes the angular distance and magnitude information etc, the stars in the image can be identified by more than one matching lastly [Scholl,1993; Bezooijen,194;padgett,etc.,1997;Douma,etc,1997;Mortari etc., 2001]. The other class is to construct an independent pattern for each star, and identify the current star by identifying the neighboring model [padgett \& Delgado, 1997]. The two classes of algorithms had been compared. [Padgett, 1997; Bittanti, 2003; Gerhard, 1997].

For autonomous star recognition algorithms, the percentage of successful identification is an important index, which is influenced by many factors, such as the recognition algorithms, the field of view (FOV), and the detection ability of star sensor, etc. The star recognition algorithm is mainly be analyzed in this paper.

If the stellar camera haven't been calibrated on-orbit or the location accuracy of star image point is low,, they will cause the accuracy of angular distance becomes low, in this case, false or redundant matching will occur, which reduces the reliability of star recognition. Based on the analysis of two classes of the algorithms above, some ideas are derived as follow. (a) Constructing a stable and unitary matching pattern may be an effective way to exclude redundant matching. (b) Compared to magnitude information, angular distance information is more accurate. if angular distance is employed as the main matching element, and the magnitude as complementary matching element, the algorithm may be more reliable.(c) for matching pattern , a master star is selected and identified firstly, based on which the neighboring stars(slave stars ) can be identified, which may accelerate the speed of identification.

Based on the matching probability algorithm, in order to improve the reliability of star recognition, the concept of the longest matching chain is further puts forward in this algorithm. The selection of the master star, directed circularity pattern construction, unitary matching and the candidate matching

\footnotetext{
${ }^{*}$ corresponding author
} 
group which has the longest chain as last matching result will improve the success rate of star recognition.

This paper briefly analyzes the flaw of the original matching probability algorithm, and then gives the design thought of the directed circularity recognition algorithm in detail. For the original algorithm, when the position error of star image point is little or the magnitude error is large, its successful identification rates and matching efficiency are higher than the matching group algorithm [Bezooijen,1994]. Consequently, the proposed algorithm is only compared with the original algorithm in the reliability and efficiency aspect in this paper, which is verified by simulated and real experiments.

\section{THE MATCHING PROBABILITY ALGORITHM}

\subsection{The Principle}

The star recognition algorithm based on matching probability firstly chooses a star in the center of the stellar image(also called the central star), and then records the appearance times of the star identification code(ID) that meet matching requirement, and lastly identify the central star by comparing the appearance times. As shown in figure 1,2 , a radial geometry pattern is constituted by linking the central star to other neighboring stars in the FOV. The Angular distance matching is implemented to identify the central star, for all "links" meeting matching criteria in the star database and the IDs of two corresponding stars are accumulated. The navigation star that has the most appearance times is regarded as the correspondence of the central star. As shown in Table 1 , the central image star whose index is 0 should be corresponding to the navigation star whose index is 14 in star catalogue. The central star identified by probability statistics can avoid the magnitude as the matching feature, so this algorithm is insensitive to magnitude error [Xie, 2008].

Once the central star is identified, the angular distance group which meets matching criteria before will be filtered. If one angular distance contains the identified central star, the other star of the angular distance may be corresponding adjacent star. As shown in table 2, if one adjacent star matches more than one candidate navigation stars, the next matching between the adjacent stars are implemented to eliminate these superfluous stars. This algorithm inherits the merit of the matching group algorithm and guarantees the high reliability.

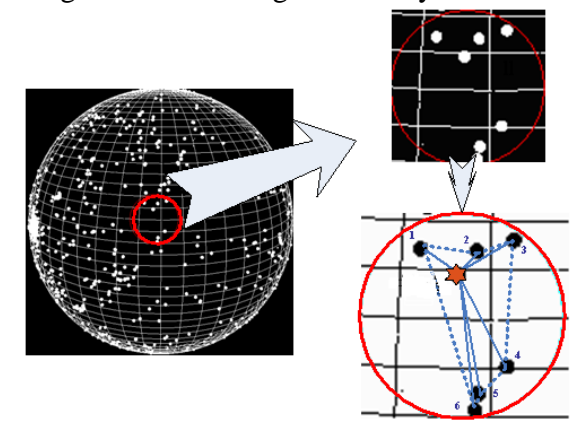

Figure.1. Star Extraction and pattern Construction.

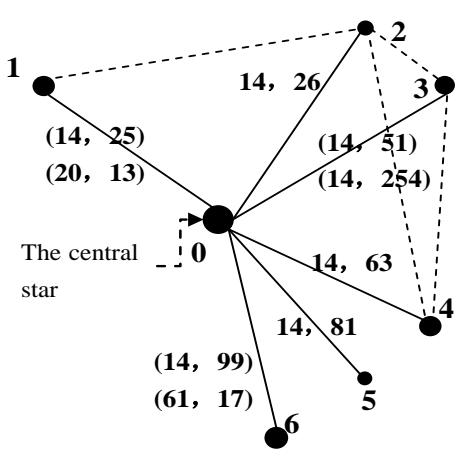

Figure.2. Central and Neighbouring Star identification.

\begin{tabular}{|cc|}
\hline $\begin{array}{c}\text { Candidate } \\
\text { navigation Star ID }\end{array}$ & $\begin{array}{c}\text { appearance } \\
\text { times }\end{array}$ \\
$\mathbf{1 4}$ & $\mathbf{7}$ \\
\hline 99 & 1 \\
61 & 1 \\
17 & 1 \\
81 & 1 \\
63 & 1 \\
51 & 1 \\
254 & 1 \\
26 & 1 \\
25 & 1 \\
20 & 1 \\
13 & 1 \\
\hline
\end{tabular}

Table 1 The statistic of Appearance times.

\begin{tabular}{|c|c|c|c|}
\hline Image & Candidate & \multicolumn{2}{|c|}{ Candidate Guide Star } \\
\hline Star & navigation & Image & navigation \\
\hline Link & star pair & Star ID & star ID \\
\hline $\mathrm{OA}$ & $\begin{array}{c}(14,99) \\
(61,17)\end{array}$ & $\mathrm{O}$ & 14 \\
\hline OB & $(14,81)$ & B & 81 \\
\hline $\mathrm{OC}$ & $(14,63)$ & $\mathrm{C}$ & 63 \\
\hline OD & $\begin{array}{l}(14,51), \\
(14,254)\end{array}$ & $\mathrm{D}$ & $\begin{array}{c}\text { Candidate: } \\
\text { 51, } 254\end{array}$ \\
\hline $\mathrm{OE}$ & $(14,26)$ & E & 26 \\
\hline OF & $\begin{array}{c}(14,25), \\
(20,13)\end{array}$ & $\mathrm{F}$ & 25 \\
\hline
\end{tabular}

Table 2 Statistic of candidate neighboring stars.

\subsection{Star Database Generation}

Since this algorithm matches the angular distance one by one according to the radicalized pattern , it need to frequently query qualified angular distance and obtain two corresponding star identification (ID) in star catalogue. Therefore, the angular distance table is stored and sorted ascendingly. As shown in Table 3, the serial number of the star in the table is noted by INDEX, and the size of angular distance is denoted by $\mathrm{I}_{12}$, and two navigation stars in star catalogue are denoted by $\mathrm{ID}_{1}$ and $\mathrm{ID}_{2}$ respectively.

When the angular distance table is founded, the angular distance that is less than the size of the FOV can be saved. In order to reduce the false matching, the navigation star should be selected before the indexed table foundation. The dissatisfying stars (the binary stars and variable stars) will be eliminated in the initial star catalogue. 


\begin{tabular}{|cccc|}
\hline INDEX & $\mathrm{I}_{12}$ & $\mathrm{ID}_{1}$ & $\mathrm{ID}_{2}$ \\
\hline.. &.. &.. &.. \\
100 & 0.108807 & 13619 & 14058 \\
101 & 0.109013 & 6063 & 5441 \\
102 & 0.109079 & 9523 & 9538 \\
$\ldots$ & $\ldots$ & $\ldots$ & $\ldots$ \\
\hline
\end{tabular}

Table 3 Angular Distance Indexed Table.

\section{A DIRECTED CIRCULARITY PATTERN ALGORITHM}

\subsection{The analysis of the original algorithm}

The original algorithm identify the central star by the statistics of matching times, which can avoid star magnitude as matching elements, so the original algorithm is insensitive to magnitude error. Once the central star is identified correctly, based on which, the adjacent stars are also identified. It accelerates the speed of star identification. It is effective when the calibration accuracy of stellar camera parameter and the position accuracy of image point are high. But in the poor condition, such as the parameters of the stellar camera aren't calibrated on-orbit, or the location accuracy of the star image points is low, some problems will be arisen, which is analyzed theoretically as follow.

\subsubsection{The Selection of the Central Star}

This algorithm selects the bright star which is close to the image center as the central star. In such case, the unsuccessful identification of the central star occurs occasionally. There are two important reasons. One is the center star can't necessarily detected by all star sensors since the different star sensors have different detection ability. The other is it doesn't assure that the central star must be in navigation star catalogue ,as the initial star catalogue is filter by VMT and other selection algorithm[Vedder,2002;Kim,1993;Samaan;2003;Zheng,2004].

\subsubsection{The Identification of the Central Star}

If the stellar camera parameters aren't calibrated or the calibration accuracy isn't high, there is major difference between the angular distance of the observable stars and corresponding catalogued stars, so the matching threshold must increase to accommodate it, which assures that the correct navigation stars can't be excluded. But in such a case, the navigation star which has most matching times doesn't necessarily correspond to the central star in the image, and then the central star is falsely identified.

\subsubsection{The Search Strategy}

This algorithm employs the k-vector method [Mortari, etc.2000], which establishes the linear relationship between the angular distance and its IDs. If the amount of the star catalog or the FOV is large, the relationship of angular distance and star index can't be described exactly only by the linear fitting, and then the lower and upper limit is difficult to determine. If the lower and upper limit is set too small, it will exclude the correct navigation star, else it will introduce excessive false stars, which decrease the matching efficiency.

\subsubsection{The Matching Strategy}

For original algorithm, if the central star is identified, based on which, other neighboring stars will be determined easily. When there are redundant navigation stars to match adjacent stars, it needs to eliminate the false items by adjacent stars matching until the unique matching group appears. The elimination process of false star is time-consuming.

\subsection{The improvement and innovation}

The novel algorithm not only inherits probability matching strategy, but also expands other ideas to improve the matching reliability. These specific improvements will be given as follows:

\subsubsection{The Brightest Star as the Master Star.}

In the original algorithm, whether "the central star" lies in the image center has little effect on the identification of the central star. On the other hand, the brightness of the star is an important index. The smaller star magnitude, the larger the probability the navigation star is included in star catalogue or the star can be detected. Therefore, the improved algorithm selects the brightest navigation star (except binary stars and variable stars), since it isn't necessarily close to the center, so it is called "the Master star" here.

\subsubsection{Search Strategy Optimization.}

The original algorithm uses $\mathrm{k}$-vector method to match the angular distance [Mortari, etc., 2000]. It is to find the angular distance by the linear fitting, which is faster than the binary tree search. However, as seen in figure 2, the angular distance and its index isn't strictly linear relationship, so it needs to piecewise fit them.

As shown in figure 3, the angular distance groups are partition by $2^{\circ}$, the linear fitting is implemented in each subsection. The black visible and dashed lines are alternately displayed, which denote different subsection. Specific formula is listed as followed. $a_{i}, b_{i}$ describe the $i$-th coefficient of angular distance groups. The search result will be more accurate and reliable based on the fitting result.

$$
\mathrm{y}=\left\{\begin{array}{c}
a_{0} x+b_{0},(0<y<1) \\
. . \\
a_{i} x+b_{i},(i-1<y<i) \\
. . \\
. \cdot
\end{array}\right.
$$

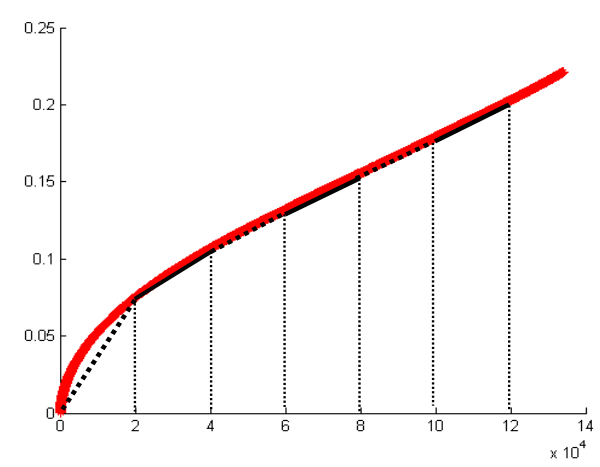

Figure. 3. The relationship between angle distance and index.

\subsubsection{Directed Circularity Pattern Construction.}

For the original algorithm, when the accuracy of angular distance is large caused by some reason as mentioned, there are many redundant matching groups, which is needed to be elinimilate one by one. The process is time-consuming and complex.

For the modified algorithm, a new image coordinate system is established, whose center is the Master star, $\mathrm{x}$ and $\mathrm{y}$ axes are parallel to that of the image center coordinates system. The separation angle of the directed vector of the Master star and adjacent star and the $\mathrm{x}$ axes can be computed, and the adjacent stars are indexed according to the separation angle, the first index is 0 . Connected directed Counterclockwise relationship will be founded. The structure of circularity 
is Basestar $\rightarrow(0 \rightarrow 1 \rightarrow 2 \rightarrow 3 \rightarrow 4 \rightarrow 5 \rightarrow 0$, the angular distance and the star index in image will be recorded as shown in table 4 .

\begin{tabular}{|c|cccc|}
\hline \multirow{6}{*}{ BaseStar } & $\begin{array}{c}\text { Angular Distance } \\
\text { Index0 }\end{array}$ & $\begin{array}{c}\text { Angular Distance } \\
\mathrm{E}_{01}\end{array}$ & $\begin{array}{c}\text { Star } \\
\text { Index0 }\end{array}$ & $\begin{array}{c}\text { Star } \\
\text { Index1 }\end{array}$ \\
\cline { 2 - 5 } & 1 & $\mathrm{E}_{12}$ & 1 & 2 \\
& 2 & $\mathrm{E}_{23}$ & 2 & 3 \\
& 3 & $\mathrm{E}_{34}$ & 3 & 4 \\
& 5 & $\mathrm{E}_{45}$ & 4 & 5 \\
& & $\mathrm{E}_{50}$ & 5 & 0 \\
\hline
\end{tabular}

Table 4 Data structure table of ring pattern.

\subsubsection{The Longest Matching Chain.}

In all candidate matching groups, matching pair between adjacent stars are needed to match one by one. As shown in figure 4, the matching result obtained is seen in figure 4. Each neighboring stars will exist multiple candidate navigation star, but it also ensures that star index are connected each other. Based on the reliability theory, the longest continuous chains are taken as the final result. It can be described by the formula (2).

$$
\begin{aligned}
& \text { Chain }_{\mathrm{k}}\left(\text { Pair }_{0}, \ldots . \text { Pair }_{\mathrm{i}}, \ldots . .\right)=\arg \max \left(\mathrm{N}\left(\text { Chain }_{\mathrm{k}}\left(\mathrm{Pair}_{0}, \ldots \text { Pair }_{\mathrm{i}}, \ldots . .\right)\right)\right) \\
& (\mathrm{i}=0, \ldots \mathrm{n} ; \mathrm{k}=0, \ldots . \mathrm{m})
\end{aligned}
$$

Chain $_{\mathrm{k}}\left(\right.$ Pair $_{0}, \ldots$ Pair $\left., \mathrm{P}_{\mathrm{i}}, \ldots ..\right)$ denotes the k-th matching chain. Pairi denotes the i-th matching pair in k-th matching chain. $\mathrm{N}\left(\right.$ Chain $_{\mathrm{k}}\left(\right.$ Pair $_{0}, \ldots$ Pair $\left.\left._{\mathrm{i}}, \ldots ..\right)\right)$ denotes the number of $\mathrm{k}$-th matching chain, $m$ denotes the number of the chain.

Additionally, if the chain fracture occurs in the process of matching, the longest chain is still considered as a final result, which can greatly increase the reliability of star identification.

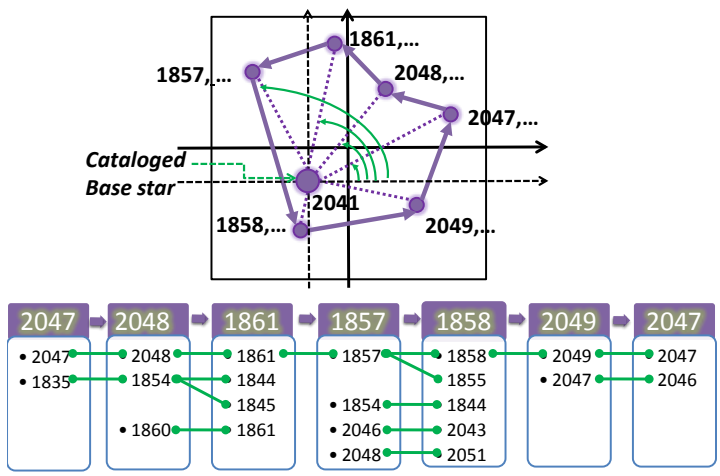

Figure.4. The scheme of matching chain.

\subsubsection{The self-adaptive adjustment of the matching threshold.}

This algorithm has high reliability, and it is also effective in the poor initial conditions, e.g. the parameter of the camera isn't calibrated. In this case, the matching threshold has to be set high, and it has the cost of bringing down the matching speed. With the improvement of the matching conditions, for example, the stellar camera are on-orbit calibrated using the identification images, the matching threshold can be set low, and the matching speed is accelerated and the reliability is improved as well.

\subsubsection{The Magnitudes as matching characteristics.}

The improved algorithm can be applicable to the conditions that the appearance magnitudes aren't able to measure or even unknown. It becomes more effective when the visual magnitudes are known. Once the appearance magnitude is accurately obtained, they are considered as the matching characteristics, and added to the angular distance table, as shown in table 5 .

\begin{tabular}{|cccccc|}
\hline INDEX & I12 & ID1 & ID2 & $\begin{array}{c}\text { MAG1 } \\
(\times 100)\end{array}$ & $\begin{array}{c}\text { MAG2 } \\
(\times 100)\end{array}$ \\
\hline & & & & & \\
$\ldots$ & $\ldots$ & $\ldots$ & $\ldots$ & $\ldots$ & $\ldots$ \\
100 & 0.108807 & 13619 & 14058 & 567 & 496 \\
101 & 0.109013 & 6063 & 5441 & 524 & 591 \\
102 & 0.109079 & 9523 & 9538 & 401 & 485 \\
$\ldots$ & $\ldots$ & $\ldots$ & $\ldots$ & $\ldots$ & $\ldots$ \\
\hline
\end{tabular}

Table 5 The star Database with magnitude information.

The MAG1and MAG2 respectively denotes the magnitudes of two stars in star catalog. As an important matching parameter, the star magnitude can be used to eliminate the false candidate matching result further. The regulation is described by formula (3). The mag1and mag2 denote two measured magnitudes of angular distance, and $\mathrm{T}_{\mathrm{E}}$ and $\mathrm{T}_{\mathrm{Mag}}$ denote angular distance and magnitude threshold.

$$
\left\{\begin{array}{c}
\left|E_{i}-E_{j}\right|<T_{E} \\
\left(\left|M A G_{1}-\operatorname{mag}_{1}\right|<T_{\text {mag }} \& \&\left|M A G_{2}-\operatorname{mag}_{2}\right|<T_{\text {mag }}\right)
\end{array}\right.
$$

\subsection{The procedure of the algorithm}

Based on the design of the longest matching chain, it needs to found the navigation angular distances table, and then calculate and save the linear fitting coefficient of angular distances and their IDs in each subsection. The star identification can be implemented after the angular distances table is constructed. The identification process is described as follows:

(1) The brightest star in the FOV is considered as the Master star. In the image pre-processing, the double stars and these very bright celestial body (such as the moon, the sun, etc.) are removed to avoid to be selected as the Master star.

(2)The Master star is treated as the starting point, and the radiant pattern is constructed by linking the staring point with the neighboring stars in the FOV.

(3)The new image coordinates are established, in which the Master star is original point. The new $\mathrm{x}$ and $\mathrm{y}$ axis is respectively parallel to the corresponding axes of the image coordinate. The adjacent stars are sorted in ascending order according to the separation angle, which is formed by the vector the current adjacent star and the original point and $\mathrm{x}$ axis, which is indexed from 0 . The stars are connected to construct a circularity pattern.

(4)Each angular distance is matched with angular distance catalogue, and the satisfied candidate navigation stars are obtained. The numbers of the repeated candidate navigation stars are recorded and are sorted descendingly. The navigation star will be chosen, whose repeated number is larger than the number of neighboring stars, while the neighboring groups of the candidate Master star will be recorded.

(5)The candidate neighboring stars of each candidate Master star are confirmed in order. The longest matching chain will be output as the final result. It is seen from figure 4, all neighboring stars are identified successfully and the matching result is 2047-2048-1861-1857-1858-2049-2047. If the chain is broken, it will not affect the final result. 
(6)Once the number of navigation stars in the longest chain equals the amount of neighboring stars, it shows that all of the neighboring stars are identified successfully, and it is the final matching results, and the matching process will exit, otherwise, all neighboring stars are matched in sequence, and the chain with most navigation stars will be the matched result.

\section{EXPERIMENT}

The star catalogue Tycho-2 (J2000) was employed for experiment, whose data types include star index, magnitude, the right ascension (hour, minute, second) and the declination (degree, arc minute, arc second) etc [The Tycho-2 Catalogue Information and Links,2008]. There are 14581 stars that brighter than $6.95 \mathrm{mv}$ in the initial star catalog. Before star database construction, the binary star and variable star should be eliminated firstly; secondly the navigation star should be selected considering the uniformity and mission requirement [Samaan, 2003; Kim, 2001; Zheng, 2004]. Then 4938 stars are selected when the upper and lower limit of magnitude is 0 and 6.0 (mv).

The parameters of the simulated camera are listed as followed. The Field of View (FOV) is $8^{\circ} \times 8^{\circ}$, the size of CCD is $512 \times 512$ pixels, and each pixel size is $13 \mathrm{um}$. The focal length is $35.6 \mathrm{~mm}$, and the principle point is 0 pixel. According to the design of algorithm, the Angular Distances Table is constructed which has 135278 items.

In this section, the original algorithm is employed for comparison, In order to compare conveniently, the accuracy unit is converted to arc second based on that the spatial accuracy of one pixel is 56.25 arc second.

The first experiment is done when the random magnitude errors range from 0.1 to $1(\mathrm{mv})$, and the interval is set $0.1 \mathrm{mv}$. The random location error is set 30 arcseconds (0.53pixel). The star image point coordinates are simulated at random optical axis orientation based on the basic star catalog; the experiment process is repeated 1000 times at different optical axis orientation. The experimental result is described by the successful identification percent in 1000 times. It is seen from figure 5 that the magnitude error has little effect on both of two algorithms. The reason is the magnitude isn't used for the star identification for them. Without doubt, If the magnitude can be measured accurately and used for the star identification, which can be used to reduce the search range, and improve the reliability of the algorithm .In addition, when the magnitude is same, the success ratios of the identification is higher than the original algorithm. The reason is the improved algorithm adopts the unitary matching strategy, which induces the false matching probability, and meanwhile, the improved algorithm has fault-tolerance, which seldom exclude candidate correct star in star identification process.

Another experiment is also done at 1000 times random optical axis orientation, when the magnitude error is $0.5(\mathrm{mv}, 3 \sigma)$, and the location error is $0,0.3,0.6,0.9,1.2,1.5,1.8,2.1$,

$2.4,2.7$ and 3.0 pixel respectively. The experimental result is shown in figure 5 . As seen from figure 6 , when the location error ranges from 0 to 1 pixel, the success identification rates of the improved algorithm is little higher than the original algorithm, and the advantage of the improved algorithm is obvious when the location error is large (achieving 1.5 3 pixel). The reason is that both of the two algorithms are dependent on the angular distance matching, and angular distance is accurate when the position error is little, so their successful identification ratios are high. But when the position error is large, the error of the angular distance is also large, and then in the process of the master star identification, the navigation star that has most repeated times isn't necessarily the actual master star. For the original algorithm, it directly considers the navigation stars with most repeat times is master star, based on which ,other neighboring stars will be identified, so once the master star isn't identified, the identification fails. For the improved algorithm, it considers all navigation stars whose repeated times which is larger than certain threshold are candidate master stars, ( the half of number of neighboring stars are used as the threshold ), which assure that the correct star can't be excluded at the beginning , and then the neighboring stars corresponding to the candidate master star are construct circularity pattern, the correct result can be obtained by unitary matching who has longest chains, which assure the identification rate .Generally, the reliability of the improved algorithm is higher than the original algorithm.

At present, there are many star centroiding algorithms, and the position accuracy of the star image points has achieved 0.1 pixel [Rufino,2003],so the improved algorithm have high practicability.

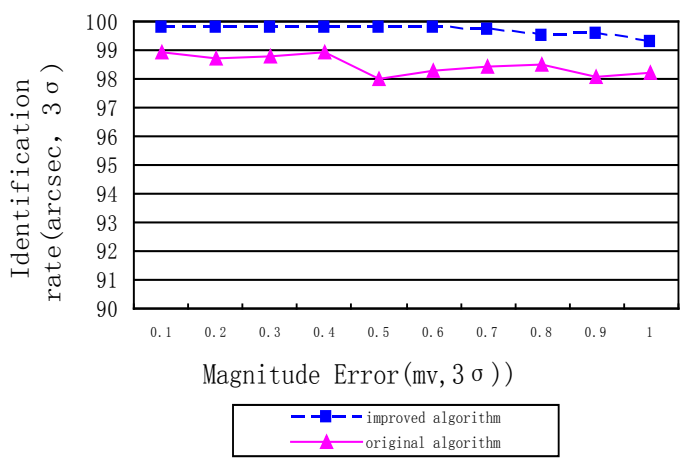

Figure.5. Success identification rates comparability under certain magnitude error and random position error.

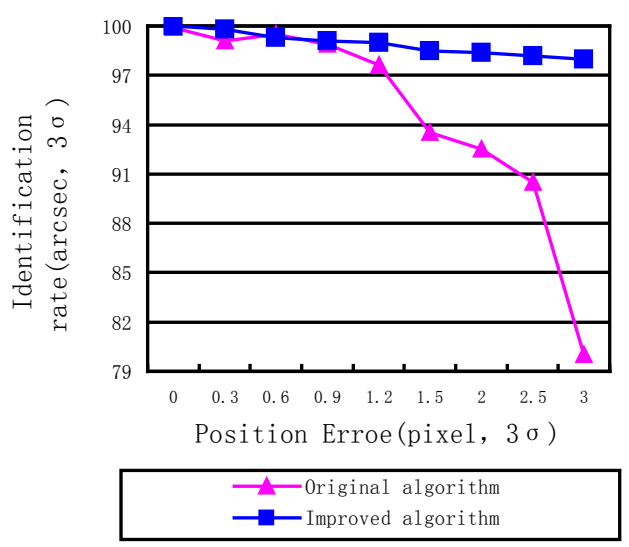

Figure.6. Success identification rates comparability under certain position error and random magnitude error.

\section{CONCLUSION}

A directed circularity pattern algorithm is elaborated in this paper. This algorithm optimizes the selection of the master star, and constructs the directed circularity pattern for adjacent stars, which are used for unitary matching, the matching group with the longest chain is considered as the matching result, these strategies are used to improve the reliability of the proposed algorithm. The simulated experiment shows that the improved 
algorithm is also insensitive to magnitude error, compared with the matching probability algorithm, and when the angular distance error is large in the poor condition, the success identification rates of the improved algorithm greatly better than the original algorithm. It is seen that the memory capacity of the algorithm is small and the efficiency of the algorithm is high by the performance analysis, which indicates the improved algorithm has practical application value for attitude determination of high resolution remote sensing satellite.

\section{REFERENCES}

[1] Wertz, J., 1978. Spacecraft Attitude Determination and Control. Dordrecht, The Netherlands: D. Reidel Publishing Company.

[2] Levine, S., Dennis, R., and Bachman, K.L., 1991. Strap down astro-inertial navigation utilizing the optical wide-angle lens star tracker, Navigation. Journal of the Institute of Navigation, 37(4), pp.347 362.

[3] Eisenma, A.R., and Liebe, C.C., 1997. The new generation of autonomous star trackers. SPIE, Vol. 3221, 524 535.

[4] Clouse, D., and Padgett, C., 2000. Small field-of-view star identification using Bayesian Decision theory. IEEE Transactions on Aerospace and Electronic Systems, 36(3):773 783.

[5] Spot image, 2005.Pre-processing levels and location accuracy, Technical information. www.spotimage.com.

[6] John Leif Jørgensen, Troelz Denver, Maurizio Betto,Pieter Van den Braembussche , THE PROBA SATELLITE STAR TRACKER PERFORMANCE

[7] Ball Aerospace\& Technologies Corp. CT-601 High Accuracy Star Tracker

[8] Doo Chun Seo, Ji Yeon Yang, Dong Han Lee, Jeong Heon Song, Hyo Suk Lim. 2008. KOMPSAT-2 DIRECT SENSOR MODELING AND GEOMETRIC CALIBRATION/VALIDATION,The International Archives of the Photogrammetry, Remote Sensing and Spatial Information Sciences. Vol. XXXVII. Part B1. Beijing

[9] Digitalglobe constellation: Quickbird Imaging Satellite, www.digitalgloble.com

[10] A. Bouillon, P. Gigord, SPOT 5 HRS LOCATION PERFORMANCE TUNING AND MONITOCIRCULARITY PRINCIPLES.

[11] Technical features of the SPOT 5 system,Press kit, pp:5 10

[12] Samaan, M. A., Pollock, T. C., and Junkins, J. L., 2002. Predictive Centroiding for Star Trackers with the Effect of Image Smear, Journal of the Astronautical Sciences, Vol. 50, 113 123.

[13] G. Rufino, D, 2003.Enhancement of the centroiding algorithm for star tracker measure refinement, Accardo/Acta Astronautica VOL 53:135 147.

[14] Shuster, M., and Oh, S.,1981.Three-axis attitude determination from vector observations.Journal of Guidance, Control and Dynamics, 4(1): 70 77.

[15] Markley,F.,1993.Attitude determination using vector observations: A fast optimal matrix algorithm. The Journal of the Astronautical Sciences, 41(2): 261 280.

[16] Mortari, D,1998.Euler-q algorithm for attitude determination from vector observations. Journal of Guidance, Control and Dynamics, 21 (2): 328 334.

[17] M.G.Leitmann, N.M.G., J.M.Rebordao,M.Fouquet,M.Sweeting, 1994. Star Imaging System:In-Flight Performance. SPIE. 2317: 167 177.

[18] C.padgett.Kreutz-Delgado,S.U.D.,1997.Evaluation of Star Identification Techniques. Journal of Guidance Control and Dynamics. 20: p. 259 267.

[19] Quine, B.M.,Durrant-Whyte,H.F.,1995.A fast autonomous star acquisition algorithm for spacecraft, In proceeding of IFAC Autonomous Control Conference, Beijing

[20] Douma,S.R.,1997.Development of an Algorithm for Autonomous Star Identification, Delft University of Technology, Faculty of Aerospace Engineecircularity.

[21] Liebe, C.C., 1995. Star Trackers for Attitude Determination, IEEE AES Systems Magazine, June.
[22] Bezooijen.RWH. v., 1994. True sky demonstration of an autonomous star tracker. SPIE, 2221: 156 168.

[23] Padgett, C., and Kreutz-Delgado, K.A,1997. grid algorithm for autonomous star identification. IEEE Transactions on Aerospace and Electronic Systems, 33(1): 202 213.

[24] Mortari D, Junkins, J L., 2001. Lost-in-space pyramid algorithm for robust star pattern recognition. 24th Annual AAS Guidance and Control Conference. Breckenridge CO, AAS Paper 01-004.

[25] Anderson, D., 1991. Autonomous Star Sensing and Pattern Recognition for Spacecraft Attitude Determination, $\mathrm{PhD}$ dissertation, Texas A\&M University, College Station, TX.

[26] E.SILANI, M.LOVERA, 2006. Star identification algorithms: novel approach \&comparison study. IEEE Transactions on Aerospace and Electronic Systems, Vol.42, NO, 4, pp: 1275-1288.

[27] MALAK A, 2005. SAMAAN, DANIELE MORTARI JOHN L. JUNKINS, Recursive Mode star Identification Algorithms. IEEE TRANSACTIONS ON AEROSPACE AND ELECTRONIC SYSTEMS. 41(4): 1246 1254.

[28] Scholl.MS, 1993. Star field identification algorithm performance verification using simulated star fields. SPIE 2019: 275 290.

[29] Hyunjae Lee, Choong-Suk Oh and Hyochoong Bang,2003. Modified Grid Algorithm for star pattern Identification by using star trackers, IEEE TRANSACTIONS ON AEROSPACE AND ELECTRONIC SYSTEMS.pp:385 391.

[30] Bittanti, S., Marchi, E. D., Giranzani, M., Lovera, M., LuubkeOssenbeck, B., and Silani, E. , 2003. A novel algorithm for fully autonomous star identification. In 13th IFAC Symposium on System Identification,Rotterdam, The Netherlands.

[31] Bittanti, S., Marchi, E. D., Giranzani, M., Lovera, M., LuubkeOssenbeck, B., and Silani, E, 2003. Star identification algorithms: A novel approach and a comparison study. In 54th International Astronautical Congress, Bremen, Germany.

[32] Gerhard, I., Junk, P., and L"ubke-Ossenbeck, B. Abrixas,1997. satellite technical concept. In 48th International Astronautical Congress, Turin, Italy.

[33] Xie Junfeng, Jiang Wanshou, Gong Jianya,2008. A New Star Identification algorithm based on matching Probability, IEEE International Geosciences And Remote Sensing Symposium, Boston, USA. Vol 3, 7-11 July 2008 P: 1166-1169(III)

[34] Mortari, D. and Neta, B. , 2000. k-vector range searching techniques, Advances in the Astronautical Sciences, Vol. 105, Pt. I, pp. 449 464.

[35] The Tycho-2 Catalogue Information and Links. http://www.astro.ku.dk/ erik/Tycho-2/, 2008-12-22

[36] Kim, H.Y., and Junkins, J.L.,2002. Self-organizing navigation star selection algorithm for star tracer: thinning method. Proc. Aerospace Conference,IEEE, Big Sky, MT, USA, pp. 2276-2283.

[37] Malak A. Samaan, Christian Bruccoleri, Daniele Mortari andJohn L. Junkins, 2003. Novel Techniques for Creating Nearly Uniform Star Catalog(AAS 03-609) Advances in the Astronautical Sciences, Part I,Vol 116,pp:1691 1704.

[38] Zheng S.,2004. Wu W R., Tian J W., Liu J. A novel regression selection algorithm of navigation star. Journal of Astronautics. 25(1).

[39] Vedder, J.D., 1993.Star trackers, star catalog, and attitude determination:probabilistic aspects of system design, J. Guid. Control Dyn., 16, (3), pp. 499 504.

[40] The Tycho-2 Catalogue Information and Links. http://www.astro.ku.dk/ erik/Tycho-2/, 2008-12-22.

[41] Jean meeus.,1991.Astronomical algorithms,Willmannbell,inc.P.O.Box 35025,Richmond,Virginia 23235.PP:129 136

\section{ACKNOWLEDGEMENTS}

This work was supported by the National Key Technology Support Program under Grant 2011BAB01B02. 